\title{
Secretary Problems via Linear Programming
}

\author{
Niv Buchbinder ${ }^{1}$, Kamal Jain ${ }^{2}$, and Mohit Singh ${ }^{3}$ \\ 1 Microsoft Research, New England, Cambridge, MA. \\ 2 Microsoft Research, Redmond, WA, USA. \\ 3 McGill University, Montreal, Canada.
}

\begin{abstract}
In the classical secretary problem an employer would like to choose the best candidate among $n$ competing candidates that arrive in a random order. This basic concept of $n$ elements arriving in a random order and irrevocable decisions made by an algorithm have been explored extensively over the years, and used for modeling the behavior of many processes. Our main contribution is a new linear programming technique that we introduce as a tool for obtaining and analyzing mechanisms for the secretary problem and its variants. The linear program is formulated using judiciously chosen variables and constraints and we show a one-toone correspondence between mechanisms for the secretary problem and feasible solutions to the linear program. Capturing the set of mechanisms as a linear polytope holds the following immediate advantages.

- Computing the optimal mechanism reduces to solving a linear program.

- Proving an upper bound on the performance of any mechanism reduces to finding a feasible solution to the dual program.

- Exploring variants of the problem is as simple as adding new constraints, or manipulating the objective function of the linear program.

We demonstrate these ideas by exploring some natural variants of the secretary problem. In particular, using our approach, we design optimal secretary mechanisms in which the probability of selecting a candidate at any position is equal. We refer to such mechanisms as incentive compatible and these mechanisms are motivated by the recent applications of secretary problems to online auctions. We also show a family of linear programs which characterize all mechanisms that are allowed to choose $J$ candidates and gain profit from the $K$ best candidates. We believe that linear programming based approach may be very helpful in the context of other variants of the secretary problem.
\end{abstract}

\section{Introduction}

In the classical secretary problem an employer would like to choose the best candidate among $n$ competing candidates. The candidates are assumed to arrive in a random order. After each interview, the position of the interviewee in the total order is revealed vis-á-vis already interviewed candidates. The interviewer has to decide, irrevocably, whether to accept the candidate for the position or 
to reject the candidate. The objective in the basic problem is to accept the best candidate with high probability. A mechanism used for choosing the best candidate is to interview the first $n / e$ candidates for the purpose of evaluation, and then hire the first candidate that is better than all previous candidates. Analysis of the mechanism shows that it hires the best candidate with probability $1 / e$ and that it is optimal $[8,18]$.

This basic concept of $n$ elements arriving in a random order and irrevocable decisions made by an algorithm have been explored extensively over the years. We refer the reader to the survey by Ferguson [9] on the historical and extensive work on different variants of the secretary problem. Recently, there has been a interest in the secretary problem with its application to the online auction problem $[13,3]$. This has led to the study of variants of the secretary problem which are motivated by this application. For example, [15] studied a setting in which the mechanism is allowed to select multiple candidates and the goal is to maximize the expected profit. Imposing other combinatorial structure on the set of selected candidates, for example, selecting elements which form an independent set of a matroid [4], selecting elements that satisfy a given knapsack constraint [2], selecting elements that form a matching in a graph or hypergraph [16], have also been studied. Other variants include when the profit of selecting a secretary is discounted with time [5]. Therefore, finding new ways of abstracting, as well as analyzing and designing algorithms, for secretary type problems is of major interest.

\subsection{Our Contributions}

Our main contribution is a new linear programming technique that we introduce as a tool for obtaining and analyzing mechanisms for various secretary problems. We introduce a linear program with judiciously chosen variables and constraints and show a one-to-one correspondence between mechanisms for the secretary problem and feasible solutions to the linear program. Obtaining a mechanism which maximizes a certain objective therefore reduces to finding an optimal solution to the linear program. We use linear programming duality to give a simple proof that the mechanism obtained is optimal. We illustrate our technique by applying it to the classical secretary problem and obtaining a simple proof of optimality of the $\frac{1}{e}$ mechanism [8] in Section 2 .

Our linear program for the classical secretary problem consists of a single constraint for each position $i$, bounding the probability that the mechanism may select the $i$ th candidate. Despite its simplicity, we show that such a set of constraints suffices to correctly capture all possible mechanisms. Thus, optimizing over this polytope results in the optimal mechanism. The simplicity and the tightness of the linear programming formulation makes it flexible and applicable to many other variants. Capturing the set of mechanisms as a linear polytope holds the following immediate advantages.

- Computing the optimal mechanism reduces to solving a linear program. 
- Proving an upper bound on the performance of any mechanism reduces to finding a feasible solution to the dual program.

- Exploring variants of the problem is as simple as adding new constraints, or manipulating the objective function of the linear program.

We next demonstrate these ideas by exploring some natural variants of the secretary problem.

Incentive Compatibility. As discussed earlier, the optimal mechanism for the classical secretary problem is to interview the first $n / e$ candidates for the purpose of evaluation, and then hire the first candidate that is better than all previous candidates. This mechanism suffers from a crucial drawback. The candidates arriving early have an incentive to delay their interview and candidates arriving after the position $\frac{n}{e}+1$ have an incentive to advance their interview. Such a behavior challenges the main assumption of the model that interviewees arrive in a random order. This issue of incentives is of major importance especially since secretary problems have been used recently in the context of online auctions $[13$, $3]$.

Using the linear programming technique, we study mechanisms that are incentive compatible. We call a mechanism for the secretary problem incentive compatible if the probability of selecting a candidate at $i^{\text {th }}$ position is equal for each position $1 \leq i \leq n$. Since the probability of being selected in each position is the same, there is no incentive for any interviewee to change his or her position and therefore the interviewee arrives at the randomly assigned position. We show that there exists an incentive compatible mechanism which selects the best candidate with probability $1-\frac{1}{\sqrt{2}} \approx 0.29$ and that this mechanism is optimal. Incentive compatibility is captured in the linear program by introducing a set of very simple constraints.

Surprisingly, we find that the optimal incentive compatible mechanism sometime selects a candidate who is worse than a previous candidate. To deal with this issue, we call a mechanism regret-free if the mechanism only selects candidates which are better than all previous candidates. We show that the best incentive compatible mechanism which is regret free accepts the best candidate with probability $\frac{1}{4}$. Another issue with the optimal incentive compatible mechanism is that it does not always select a candidate. In the classical secretary problem, the mechanism can always pick the last candidate but this solution is unacceptable when considering incentive compatibility. We call a mechanism must-hire if it always hires a candidate. We show that there is a must-hire incentive compatible mechanism which hires the best candidate with probability $\frac{1}{4}$. All the above results are optimal and we use the linear programming technique to derive the mechanisms as well as prove their optimality.

In subsequent work [6], we further explore the importance of incentive compatibility in the context of online auctions. In this context, bidders are bidding for an item and may have an incentive to change their position if this may increase their utility. We show how to obtain truthful mechanisms for such settings 
using underlying algorithms for secretary type problems. While there are inherent differences in the auction model and the secretary problem, a mechanism for the secretary problem is used as a building block for obtaining an incentive compatible mechanism for the online auction problem.

The J-choice, K-best Secretary Problem. Our LP formulation approach is able to capture a much broader class of secretary problems. We define a most general problem that we call the $J$-Choice, $K$-best secretary problem, referred to as the $(J, K)$-secretary problem. Here, $n$ candidates arrive randomly. The mechanism is allowed to pick up to $J$ different candidates and the objective is to pick as many from the top $K$ ranked candidates. The $(1,1)$-secretary problem is the classical secretary problem. For any $J, K$, we provide a linear program which characterizes all mechanisms for the problem by generalizing the linear program for the classical secretary problem.

A sub-class that is especially interesting is the $(K, K)$-secretary problem, since it is closely related to the problem of maximizing the expected profit in a cardinal version of the problem. In the cardinal version of the problem, $n$ elements that have arbitrary non-negative values arrive in a random order. The algorithm is allowed to pick at most $k$ elements and its goal is to maximize its expected profit. We define a monotone mechanism to be an mechanism that at any position does not select an element that is $t$ best so far with probability higher than an element that is $t^{\prime}<t$ best so far. We note that any reasonable mechanism (and in particular the optimal mechanism) is monotone. The following is a simple observation. We omit the proof due to lack of space.

Observation 1 Let Alg be a monotone mechanism for the $(K, K)$-secretary problem that is c-competitive. Then the mechanism is also c-competitive for maximizing the expected profit in the cardinal version of the problem.

Kleinberg [15] gave an asymptotically tight mechanim for the cardinal version of the problem. However, this algorithm is randomized, and also not tight for small values of $k$. Better mechanisms, even restricted to small values of $k$, are helpful not only for solving the original problem, but also for improving algorithms that are based upon them. For example, the secretary knapsack algorithm [2] uses an algorithm that is $1 / e$ competitive for maximizing the expected profit for small values of $k(k \leq 27)$. Analyzing the LP asymptotically for any value $n$ is a challenge even for small value $k$. However, using our characterization we solve the problem easily for small values $k$ and $n$ which gives an idea on how competitive ratio behaves for small values of $k$. Our results appear in Table 1. We also give complete asymptotic analysis for the cases of $(1,2),(2,1)$-secretary problems.

\subsection{Related Work}

The basic secretary problem was introduced in a puzzle by Martin Gardner [11]. Dynkin [8] and Lindley [18] gave the optimal solution and showed that no other 
Number of elements allowed to be picked by the algorithm|Competitive ratio

\begin{tabular}{l|c}
\hline 1 & $1 / e=0.368$ \\
2 & 0.474 \\
3 & 0.565 \\
4 & 0.613
\end{tabular}

Table 1. Competitive ratio for Maximizing expected profit. Experimental results for $n=100$.

strategy can do better (see the historical survey by Ferguson [9] on the history of the problem). Subsequently, various variants of the secretary problem have been studied with different assumptions and requirements [20](see the survey [10]).

More recently, there has been significant work using generalizations of secretary problems as a framework for online auctions $[15,13,2,4,3]$. Incentives issues in online mechanisms have been studied in several models $[17,13,1]$. These works designed mechanisms where incentive issues were considered for both value and time strategies. For example, Hajiaghayi et. al. [13] studied a limited supply online auction problem, in which an auctioneer has a limited supply of identical goods and bidders arrive and depart dynamically. In their problem bidders also have a time window which they can lie about.

Our linear programming technique is similar to the technique of factor revealing linear programs that have been used successfully in many different settings $[12,14,19,7]$. Factor revealing linear program formulates the performance of an algorithm for a problem as a linear program (or sometimes, a more general convex program). The objective function is the approximation factor of the algorithm on the problem. Thus solving the linear program gives an upper bound on the worst case instance which an adversary could choose to maximize/minimize the approximation factor. Our technique, in contrast, captures the information structure of the problem itself by a linear program. We do not apriori assume any algorithm but formulate a linear program which captures every possible algorithm. Thus optimizing our linear program not only gives us an optimal algorithm, but it also proves that the algorithm itself is the best possible.

\section{Introducing the Technique: Classical secretary (and variants)}

In this section, we give a simple linear program which we show characterizes all possible mechanisms for the secretary problem. We stress that the LP captures not only thresholding mechanisms, but any mechanism including probabilistic mechanisms. Hence, finding the best mechanism for the secretary problem is equivalent to finding the optimal solution to the linear program. The linear program and its dual appear in Figure 1. The following two lemmas show that the linear program exactly characterizes all feasible mechanisms for the secretary problem. 


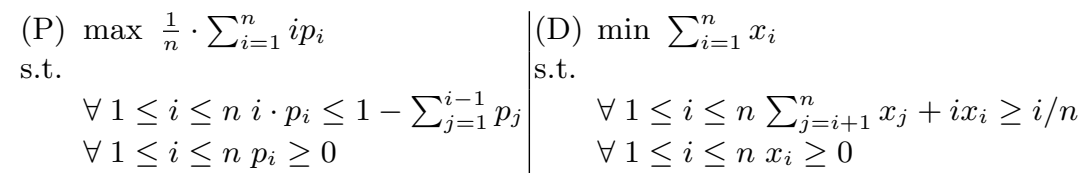

Fig. 1. Linear program and its Dual for the secretary problem

Lemma 1. (Mechanism to LP solution) Let $\pi$ be any mechanism for selecting the best candidate. Let $p_{i}^{\pi}$ denote the probability of selecting the candidate at position $i$. Then $p^{\pi}$ is a feasible solution to the linear program $(P)$, i.e, it satisfies the constraints $p_{i}^{\pi} \leq \frac{1}{i}\left(1-\sum_{j<i} p_{j}^{\pi}\right)$ for each $1 \leq i \leq n$. Moreover the objective value $\frac{1}{n} \sum_{i=1}^{n} i p_{i}^{\pi}$ is at least the probability of selecting the best candidate by $\pi$.

Proof. Let $p_{i}^{\pi}$ be the probability in which mechanism $\pi$ selects candidate $i$. Any mechanism cannot increase its chances of hiring the best candidate by selecting a candidate that is not the best so far, therefore we may consider only such mechanisms. We now show that $p^{\pi}$ satisfies the constraints of linear program.

$p_{i}^{\pi}=\operatorname{Pr}[\pi$ selects candidate $i \mid$ candidate $i$ is best so far $]$

$$
\begin{array}{r}
\cdot \operatorname{Pr}[\text { candidate } i \text { is best so far }] \\
\leq \operatorname{Pr}[\pi \text { did not select candidates }\{1, \ldots, i-1\}] \mid \text { candidate } i \text { is best so far }] \cdot \frac{1}{i}
\end{array}
$$

However, the probability of selecting candidates 1 to $i-1$ depends only on the relative ranks of these candidates and is independent on whether candidate $i$ is best so far (which can be determined after the mechanism have done its choices regarding candidates 1 to $i-1)$. Therefore, we obtain $p_{i}^{\pi} \leq \frac{1}{i}\left(1-\sum_{j<i} p_{j}^{\pi}\right)$, which proves our claim.

Now we show that the objective function of the linear program is at least the probability with which $\pi$ accepts the best candidate. Since the algorithm cannot distinguish whether the $i^{\text {th }}$ candidate is the best candidate so far or best candidate over all, the probability that the mechanism hires candidate $i$ given that the best candidate is in the $i$ th position equals the probability the mechanism hires candidate $i$ given that the best candidate among candidates 1 to $i$ is in the $i$ th position. Since the $i$ th candidate is best so far with probability $1 / i$, the latter probability is at least $i p_{i}^{\pi}$. Summing over all $n$ positions we get that $\pi$ hires the best candidate with probability at least $\frac{1}{n} \sum_{i=1}^{n} i p_{i}^{\pi}$.

Lemma 1 shows that the optimal solution to $(\mathrm{P})$ is an upper-bound on the performance of the mechanism. The following lemma shows that every LP solution actually corresponds to a mechanism which performs as well as the objective value of the solution.

Lemma 2. (LP solution to Mechanism) Let $p_{i}$ for $1 \leq i \leq n$ be any feasible LP solution to $(P)$. Then consider the mechanism $\pi$ which selects the candidate $i$ with probability $\frac{i p_{i}}{\left(1-\sum_{j<i} p_{j}\right)}$ if candidate $i$ is the best candidate so far and 


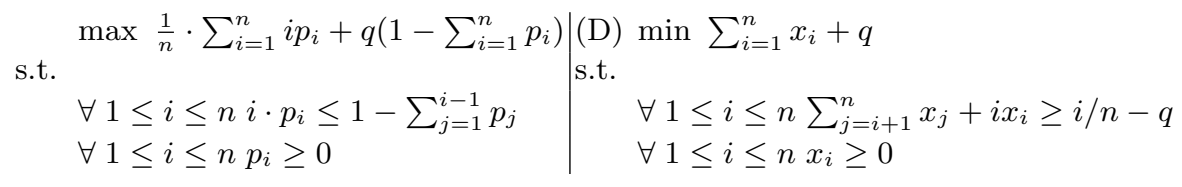

Fig. 2. Linear program and its Dual for the rehiring secretary problem

candidate $1, \ldots, i-1$ have not been selected, i.e., the mechanism reaches candidate $i$. Then $\pi$ is a mechanism which selects the best candidate with probability $\frac{1}{n} \sum_{i=1}^{n} i p_{i}$.

Proof. First, notice that the mechanism is well defined since for any $i$, $\frac{i p_{i}}{\left(1-\sum_{j<i} p_{j}\right)} \leq 1$. We prove by induction that the probability that the mechanism selects candidate at position $i$ is exactly $p_{i}$. The base case is trivial. Assume this is true until $i-1$. At step $i$, the probability we choose $i$ is the probability that we didn't choose candidates 1 to $i-1$ which is $1-\sum_{j<i} p_{j}$ times the probability that the current candidate is best so far which is $1 / i$ times $\frac{i p_{i}}{\left(1-\sum_{j<i} p_{j}\right)}$ which is exactly $p_{i}$.

The probability of hiring the $i$ th candidate given that the $i$ th candidate is the best candidate is equal the probability of hiring the $i$ th candidate given the $i$ th candidate is the best candidate among candidates 1 to $i$. Otherwise, it means that the mechanism is able to distinguish between the event of seeing the relative ranks and the absolute ranks which is a contradiction to the definition of the secretary problem. Since the $i$ th candidate is best so far with probability $1 / i$, the latter probability equals $i p_{i}$ (the mechanism hires only the best candidate so far). Summing over all possible position $n$ we get that the mechanism $\pi$ hires the best candidate with probability $\frac{1}{n} \sum_{i=1}^{n} i p_{i}$.

Using the above equivalence between LP solutions and the mechanisms, it is easy to show that the optimal mechanism can hire the best candidate with probability of no more than $1 / e$. The proof is simply by constructing a feasible solution to the dual linear program.

Lemma 3 ([8]). No mechanism can hire the best candidate with probability better than $1 / e+o(1)$.

Proof. To prove an upper bound of $1 / e$ we only need to construct a feasible dual solution to program (D) with value $1 / e$. Set $x_{i}=0$ for each $1 \leq i \leq \frac{n}{e}$ and $x_{i}=\frac{1}{n}\left(1-\sum_{j=i}^{n-1} \frac{1}{j}\right)$ for $\frac{n}{e}<i \leq n$. A simple calculation shows that $x$ is feasible and has objective value $\frac{1}{e}$.

\subsection{Allowed to Rehire}

One natural extension of the secretary problem is the case when one is allowed to rehire the best secretary at the end with certain probability. That is, suppose 


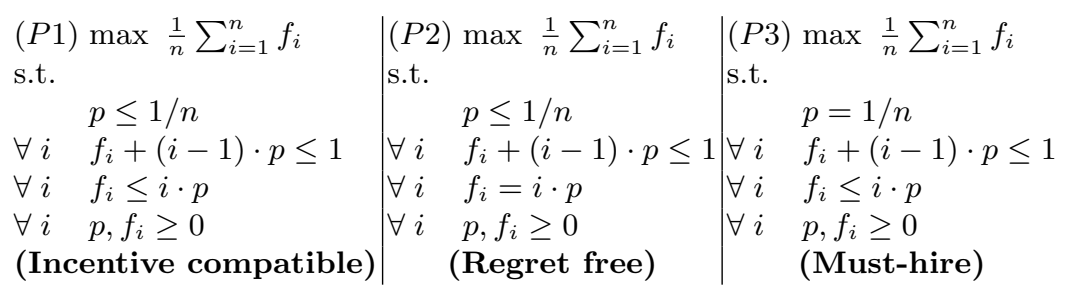

Fig. 3. (P1): Characterizes any incentive compatible mechanism. (P2) characterizes mechanisms that are regret free. (P3) characterizes mechanisms that are must-hire mechanisms.

that after the interviewer has seen all $n$ candidates, he is allowed to hire the best candidate with certain probability $q$ if no other candidate has been hired. Observe that if $q=0$, the problem reduces to the classical secretary problem while if $q=1$, then the optimal strategy is to wait till the end and then hire the best candidate. We give a tight description of strategies as $q$ changes. This can be achieved simply by modifying the linear program: simply add in the objective function $q\left(1-\sum_{i=1}^{n} p_{i}\right)$. That is, if the mechanism did not hire any candidate you may hire the best candidate with probability $q$. Solving the primal and the corresponding dual (appear in Figure 2) give the following tight result. The proof is omitted.

Theorem 2. There is a mechanism for the rehire variant that selects the best secretary with probability $e^{-(1-q)}$ and it is optimal.

\section{Incentive Compatibility}

In this section we study incentive compatible mechanisms for the secretary problem. We design a set of mechanisms $\mathcal{M}_{p}$ and show that with certain parameters these mechanisms are the optimal mechanisms for certain secretary problems. To this end, we derive linear formulations that characterize the set of possible incentive compatible mechanisms and also analyze the dual linear programs.

The basic linear formulation that characterizes all incentive compatible mechanisms appears in Figure 3. We give a set of three linear formulations. The formulation $(P 1)$ characterizes all mechanisms that are incentive compatible, $(P 2)$ captures mechanisms that are also regret free and $(P 3)$ captures mechanisms that are must-hire mechanisms. This is formalized in the following two lemmas.

Lemma 4. (Mechanism to LP solution) Let $\pi$ be any mechanism for selecting the best candidate that is incentive compatible. Let $p^{\pi}$ denote the probability the mechanism selects a candidate at each position $i$, and let $f_{i}^{\pi}$ be the probability the mechanism selects the candidate at position $i$ given that the candidate at position $i$ is the best candidate. Then:

$-p^{\pi}, f_{i}^{\pi}$ is a feasible solution to the linear program $(P 1)$. 
- If the mechanism is also regret free then $p^{\pi}, f_{i}^{\pi}$ is a feasible solution to the linear program (P2).

- If the mechanism is also must-hire then $p^{\pi}, f_{i}^{\pi}$ is a feasible solution to the linear program (P3).

- The objective value $\frac{1}{n} \sum_{i=1}^{n} f_{i}^{\pi}$ is at least the probability of selecting the best candidate by $\pi$.

Proof. The proof follows the same ideas as in the proof of Lemma 1. The condition of incentive compatibility implies that $p_{i}=p_{j}=p$ for any two positions $i$ and $j$.

Also, in the original secretary problem, every mechanism could be modified to be a regret free mechanism. This is not true for an incentive compatible mechanism. Indeed, we have the following constraint, $f_{i} \leq i p_{i}$ since the probability of hiring in the $i$ th position is at least the probability of hiring in the $i$ th position given that the candidate is best so far times $1 / i$. If the mechanism is also supposed to be regret free then equality must hold for each $i$. In the must-hire part we demand that the sum of $p_{i}$ is 1 . The resulting formulation given in Figure 3 is after simplification.

Lemma 4 shows that the optimal solution to the linear formulations is an upper-bound on the performance of the mechanism. To show the converse we define a family of mechanisms that are defined by their probability of selecting a candidate at each position $0 \leq p \leq 1 / n$, we show that the set of feasible solutions to $(P 1)$ corresponds to the set of mechanisms $\mathcal{M}_{p}$ defined here.

\section{Incentive Compatible Mechanism $\mathcal{M}_{p}$ :}

- Let $0 \leq p \leq 1 / n$. For each $1 \leq i \leq n$, while no candidate is selected, do

- If $1 \leq i \leq \frac{1}{2 p}$, select the $i^{t h}$ candidate with probability $\frac{i}{1 / p-i+1}$ if she is the best candidate so far.

- If $\frac{1}{2 p}<i \leq n$, let $r=\frac{i}{1 / p-i+1}$. Select the $i^{\text {th }}$ candidate with probability 1 if her rank is in top $\lfloor r\rfloor$ and with probability $r-\lfloor r\rfloor$ if her rank is $\lfloor r\rfloor+1$.

The following lemma shows that every LP solution to $(P 1)$ corresponds to a mechanism which performs as well as the objective value of the solution.

Lemma 5. (LP solution to Mechanism) Let $p, f_{i}$ for $1 \leq i \leq n$ be a feasible $L P$ solution to $(P 1)$. Then the mechanism $\mathcal{M}_{p}$ selects the best candidate with probability which is at least $\frac{1}{n} \sum_{i=1}^{n} f_{i}$.

Proof. For any $p$, the optimal values of $f_{i}$ are given by the following. $f_{i}=i p$ for $1 \leq i \leq \frac{1}{2 p}$ and $f_{i}=1-(i-1) p$ for $i>\frac{1}{2 p}$. For ease of calculations, we ignore the fact the fractions need not be integers. These are exactly the values achieved by the mechanism $\mathcal{M}_{p}$ for any value $p$.

Lemma 6. The mechanism $\mathcal{M}_{p}$ is incentive compatible for each $0 \leq p \leq 1 / n$ and has efficiency of $1-\left(\frac{1}{4 p n}+\frac{p n}{2}\right)$. 
Proof. We prove by induction that the mechanism $\mathcal{M}_{p}$ selects each position $i$ with probability $p$. It is easy to verify that for $i=1$ this is true. For $i>1$. The probability the mechanism chooses position $i$ is by our induction hypothesis:

$$
r \cdot \frac{1}{i} \cdot(1-(i-1) p)=\frac{i}{1 / p+i-1} \frac{1}{i}(1-(i-1) p)=\frac{1-(i-1) p}{1 / p+i-1}=p
$$

The probability the mechanism selects the best candidate is related to $f_{i}$. $f_{i}=i p$ for $1 \leq i \leq 1 / 2 p$, and $f_{i}=1-(i-1) p$ for $1 / 2 p<i \leq n$. Thus, we get:

$$
\frac{1}{n} \sum_{i=1}^{n} f_{i}=\frac{1}{n}\left(\sum_{i=1}^{1 / 2 p} i p+\sum_{i=1 / 2 p+1}^{n}(1-(i-1) p)\right)=1-\left(\frac{1}{4 p n}+\frac{p n}{2}\right)
$$

Optimizing the linear programs $(P 1),(P 2)$ and $(P 3)$ exactly, we get the following theorem. The optimality of the mechanisms can also be shown by exhibiting an optimal dual solution.

Theorem 3. The family of mechanisms $\mathcal{M}_{p}$ achieves the following.

1. Mechanism $\mathcal{M}_{1 / \sqrt{2} n}$ is incentive compatible with efficiency of $1-\frac{1}{\sqrt{2}} \approx 0.29$.

2. Mechanism $\mathcal{M}_{1 / 2 n}$ is incentive compatible and regret free with efficiency $\frac{1}{4}$.

3. Mechanism $\mathcal{M}_{1 / n}$ is incentive compatible and must-hire with efficiency $\frac{1}{4}$.

Moreover, all these mechanism are optimal for efficiency along with the additional property.

\section{The $J$-choice $K$-best secretary problem}

In this section we study a general problem of selecting as many of the top $1, \ldots, K$ ranked secretaries given $J$ rounds to select. The mechanism is given $J$ possible rounds in which it may select a candidate, and it gains from selecting any of the first $K$ ranked candidates. The classical secretary problem is exactly 1-choice 1-best secretary problem. Other special cases include cases in which the algorithm is given $J$ rounds and get profit only for the best candidate, or getting a single round, but receive profit for any of the best $K$ candidates. Our result is a simple linear formulation that characterize all strategies for selecting the candidates.

The following two lemmas show that the above linear program exactly characterizes all feasible mechanisms for the $(J, K)$-secretary problem.

Lemma 7. (Mechanism to LP solution) Let $\pi$ be any mechanism for selecting the $(J, K)$-secretary problem. Let

$-p_{i}^{j}(\pi)$ : The probability of accepting the candidate at ith position in the $j$ th round for each $1 \leq i \leq n$ and each $1 \leq j \leq J$. 


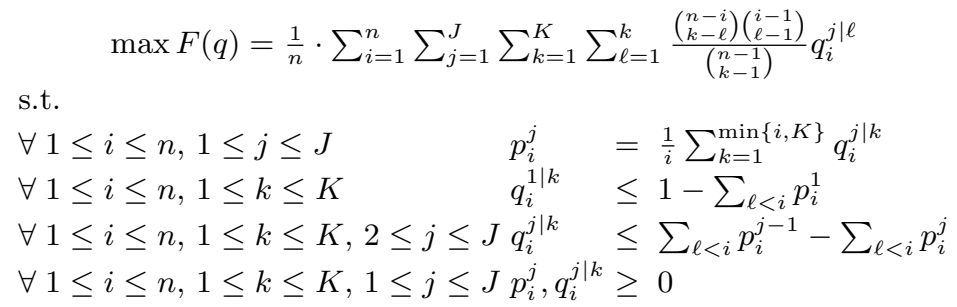

Fig. 4. Linear program for the $(J, K)$-secretary problem

$-q_{i}^{j \mid k}(\pi)$ : The probability of accepting the candidate ith position in the $j$ th round given that the candidate is the $k$ th best candidate among the $i$ first candidates for each $1 \leq i \leq n, 1 \leq j \leq J$ and $1 \leq k \leq K$.

Then $(p(\pi), q(\pi))$ is a feasible solution and expected number of top $K$ candidates selected is at most $F(p(\pi), q(\pi))$.

Proof. Let us prove the first type of constraints of the form: $p_{i}^{j}=\frac{1}{i} \sum_{k=1}^{\min \{i, K\}} q_{i}^{j \mid k}$

It is clear that there is no reason for any mechanism to select a candidate which is not at least the $K$ best so far. Such a candidate cannot be even potentially one of the $K$ best globally and therefore is not profitable for the mechanism. Therefore, we may consider only such mechanisms. Thus, the probability any mechanism selects the $i$ th candidate in the $j$ th round is the sum of the probability of selecting the $i$ th candidate in the $j$ th round given that the candidate is the $k$ th best candidate so far times $1 / i$, which is the probability that the candidate is the $k$ th best so far. We sum until the minimum between $i$ and $K$ to get the desired equality which holds for every mechanism.

Let us now prove the third type of constraints (the second type follows by the same arguments). Consider any mechanism and some position $i$ and some rounds $j$.

$q_{i}^{j \mid k}=\operatorname{Pr}[\pi$ selects candidate $i$ in round $\mathrm{j} \mid$ candidate $i$ is $k$ th best so far $]$

$\leq \operatorname{Pr}[\pi$ selects exactly $j-1$ candidates out of cand. $\{1, \ldots, i-1\}]$

| candidate $i$ is $k$ th best so far]

$=\operatorname{Pr}[\pi$ selects exactly $j-1$ candidates out of cand. $\{1, \ldots, i-1\}]$

$=\sum_{\ell<i} p_{i}^{j-1}(\pi)-\sum_{\ell<i} p_{i}^{j}(\pi)$

The inequality follows since in order to select candidate $i$ in round $j$ the mechanism must have selected exactly $j-1$ candidates out of the previous $i-1$ candidates. The following equality then follows since the decisions made by the policy with respect to the $i-1$ candidates depend only on the relative ranks of the $i-1$ candidates, and is independent of the rank of the $i$ th candidate with 
respect to these candidates. The final equality follows since the event of selecting $j-1$ candidates contains the event of selecting $j$ candidates, which concludes our proof.

Finally, let us consider the objective function and prove that it upper bounds the performance of the mechanism. For analysis purpose let us consider the probabilities $f_{i}^{j \mid k}$ that are defined as selecting the $i$ th candidate in the $j$ th round given that the $k$ th best candidate is in the $i$ th position. Note that the main difference between $f_{i}^{j \mid k}$ and $q_{i}^{j \mid k}$ is that while the former consider the $k$ th best candidate overall, the latter only looks from the algorithm's perspective and therefore looks at the event of the $k$ best candidate among the first $i$ candidates. It is easy to state the objective function using the first set of variables as simply the sum over all values of $i, j$ and $k$ of $f_{i}^{j \mid k}$ divided by $1 / n$.

To finish we simply define each $f_{i}^{j \mid k}$ in terms of $q_{i}^{j \mid k}$ which proves the lemma. Claim. For each $1 \leq i \leq n, 1 \leq j \leq J$ and $1 \leq k \leq K$, we must have

$$
f_{i}^{j \mid k}=\sum_{\ell=1}^{k} \frac{\left(\begin{array}{c}
i-1 \\
l-1
\end{array}\right)\left(\begin{array}{l}
n-i \\
k-\ell
\end{array}\right)}{\left(\begin{array}{l}
n-1 \\
k-1
\end{array}\right)} q_{i}^{j \mid \ell}
$$

The proof is omitted due to lack of space. The proof of Lemma 7 follows directly from the claim.

Lemma 7 shows that the optimal solution to $(\mathrm{P})$ is an upper-bound on the performance of the mechanism. The following lemma shows that every LP solution actually corresponds to a mechanism which performs as well as the objective value of the solution.

Lemma 8. (LP solution to Mechanism) Let $(p, q)$ be any feasible LP solution to $(P)$. Then consider the mechanism $\pi$ defined inductively as follows. For each position $1 \leq i \leq n$,

- If the mechanism has not selected any candidate among position $\{1, \ldots, i-1\}$ and the rank of candidate $i$ among $\{1, \ldots, i\}$ is $k$ for some $1 \leq k \leq K$, then select candidate $i$ with probability $\frac{q_{i}^{1 \mid k}}{1-\sum_{\ell<i} p_{i}^{1}}$.

- If the mechanism has selected $j-1$ candidates in positions $1, \ldots, i-1$ for some $2 \leq j \leq J$ and the rank of candidate $i$ among $\{1, \ldots, i\}$ is $k$ for some $1 \leq k \leq K$, then select candidate $i$ with probability $\frac{q_{i}^{j \mid k}}{\sum_{\ell<i} p_{i}^{j-1}-\sum_{\ell<i} p_{i}^{j}}$.

- Else do not select candidate $i$.

Then expected number of top $k$ candidates selected by $\pi$ is exactly $F(p, q)$.

Proof (Sketch). The proof is by induction on the steps of the algorithm. It can be verified easily that the procedure above keeps by induction that $p_{i}^{j}(\pi)=$ $p_{i}^{j}, q_{i}^{j \mid k}(\pi)=q_{i}^{j \mid k}$. That is, the probability the mechanism selects the $i$ th candidate in the $j$ th round is the same as the LP. As stated in Lemma 7 there is a correspondence between the values of $q_{i}^{j \mid k}(\pi)$ and $f_{i}^{j \mid k}(\pi)$ which is the probabilities of hiring the $i$ th candidate in the $j$ th round given that the candidate is the $k$ th best. Thus, the objective function of $\pi$ is exactly $F(p, q)$. 
We now give optimal mechanism for the $(1,2)$ and $(2,1)$-secretary problem. Observe that $(1,1)$-secretary problem is the traditional secretary problem.

Theorem 4. There exists mechanisms which achieve a performance of

1. $\frac{1}{e}+\frac{1}{e^{1.5}} \simeq 0.591$ for $(2,1)$-secretary problem.

2. $\simeq 0.572284$ for the $(1,2)$ secretary problem.

Moreover all these mechanisms are (nearly) optimal for their corresponding problems.

Proof. (Sketch) To give a mechanism, we will give a primal solution to $L P(J, K)$. The optimality is shown by exhibiting a dual solution of the same value. Due to lack of space we only prove the $(2,1)$ case.

$(2,1)$-secretary. Let $t_{1}=\frac{n}{e^{3 / 2}}$ and $t_{2}=\frac{n}{e}$. Consider the following mechanism that selects the $i^{t h}$ candidate if $i^{t h}$ candidate is best so far and $t_{1} \leq i<t_{2}$ and no other candidate has been selected or if $t_{2} \leq i \leq n$ and $i^{t h}$ candidate is best so far and at most one candidate has been selected. The performance of this mechanism is $\frac{1}{e}+\frac{1}{e^{\frac{3}{2}}}$. The mechanism corresponds to the primal LP solution where $p_{i}^{1}=0$ for $1 \leq i<t_{1}$ and $p_{i}^{1}=\frac{t_{1}-1}{i(i-1)}$ for $t_{1} \leq i \leq n, p_{i}^{2}=0$ for $1 \leq i<t_{2}$

and $p_{i}^{2}=\frac{t_{2}-t_{1}}{i(i-1)}-\frac{1}{i(i-1)} \sum_{j=1}^{i-1} \frac{t_{1}-1}{i-1}$ for $t_{2} \leq i \leq n, q_{i}^{j \mid 1}=i \cdot p_{i}^{j}$ for each $1 \leq j \leq 2$ and $1 \leq i \leq n$.

\section{Further Discussion}

Characterizing the set of mechanisms in secretary type problems as a linear polytope possess many advantages. In contrast to methods of factor revealing LPs in which LPs were used to analyze a single algorithm, here we are able to characterize all algorithms. One direction for future research is trying to capture more complex settings of a more combinatorial nature. One such example is the clean problem studied in [4] in which elements of a matroid arrive one-by-one. This problem seems extremely appealing since matroid constraints are exactly captured by a linear program. Another promising direction is obtaining upper bounds. While the linear program which characterizes the performance may be too complex to obtain a simple mechanism, the dual linear may still be used for obtaining upper bounds on the performance of any linear program. We believe that linear programming and duality is a powerful approach for studying secretary problems and will be applicable in more generality.

\section{References}

1. Baruch Awerbuch, Yossi Azar, and Adam Meyerson. Reducing Truth-Telling Online Mechanisms to Online Optimization. In In Proc. ACM Symposium on Theory of Computing, pages 503-510, 2003. 
2. M. Babaioff, N. Immorlica, D. Kempe, and R. Kleinberg. A Knapsack Secretary Problem with Applications. In Proceedings of 10th International Workshop on Approximation Algorithms for Combinatorial Optimization Problems (APPROX), 2007.

3. M. Babaioff, N. Immorlica, D. Kempe, and R. Kleinberg. Online Auctions and Generalized Secretary Problems. SIGecom Exchange, 7:1-11, 2008.

4. M. Babaioff, N. Immorlica, and R. Kleinberg. Matroids, Secretary Problems, and Online Mechanisms. In Proceedings 18th ACM-SIAM Symposium on Discrete Algorithms, 2007.

5. Moshe Babaioff, Michael Dinitz, Anupam Gupta, Nicole Immorlica, and Kunal Talwar. Secretary problems: weights and discounts. In SODA '09: Proceedings of the Nineteenth Annual ACM -SIAM Symposium on Discrete Algorithms, pages 1245-1254, Philadelphia, PA, USA, 2009. Society for Industrial and Applied Mathematics.

6. N. Buchbinder, M. Singh, and K. Jain. Incentives in Online Auctions and Secretary Problems via Linear Programming. In Manuscript, 2009.

7. Niv Buchbinder, Kamal Jain, and Joseph (Seffi) Naor. Online primal-dual algorithms for maximizing ad-auctions revenue. In Proceedings of the 15th Annual European Symposium, pages 253-264, 2007.

8. E. B. Dynkin. The Optimum Choice of the Instant for Stopping a Markov Process. Sov. Math. Dokl., 4, 1963.

9. T. S. Ferguson. Who Solved the Secretary Problem? Statist. Sci., 4:282-289, 1989.

10. P. R. Freeman. The Secretary Problem and its Extensions: A Review. International Statistical Review, 51:189-206, 1983.

11. M. Gardner. Mathematical Games. Scientific American, pages 150-153, 1960.

12. Michel Goemans and Jon Kleinberg. An improved approximation ratio for the minimum latency problem. In SODA '96: Proceedings of the seventh annual ACMSIAM symposium on Discrete algorithms, pages 152-158, 1996.

13. M. T. Hajiaghayi, R. Kleinberg, and D. C. Parkes. Adaptive Limited-Supply Online Auctions. In Proceedings of the 5th ACM Conference on Electronic Commerce, 2004.

14. Kamal Jain, Mohammad Mahdian, Evangelos Markakis, Amin Saberi, and Vijay V. Vazirani. Greedy facility location algorithms analyzed using dual fitting with factor-revealing lp. J. ACM, 50(6):795-824, 2003.

15. R. Kleinberg. A Multiple-Choice Secretary Algorithm with Applications to Online Auctions. In Proceedings of the Sixteenth Annual ACM-SIAM Symposium on Discrete algorithms, 2005.

16. Nitish Korula and Martin Pál. Algorithms for secretary problems on graphs and hypergraphs. In ICALP (2), pages 508-520, 2009.

17. Ron Lavi and Noam Nisan. Competitive Analysis of Incentive Compatible On-line Auctions. In In Proc. 2nd ACM Conf. on Electronic Commerce, pages 233-241, 2000.

18. D. V. Lindley. Dynamic Programming and Decision Theory. Applied Statistics, 10:39-51, 1961.

19. Aranyak Mehta, Amin Saberi, Umesh Vazirani, and Vijay Vazirani. Adwords and generalized online matching. J. ACM, 54(5):22, 2007.

20. Stephen M. Samuels. Secretary Problems. In Handbook of Sequential Analysis, volume 118, pages 381-405, 1991. 